\title{
Observations of circumstellar disks with infrared interferometry
}

\author{
R L Akeson \\ Michelson Science Center, California Institute of Technology, Pasadena, CA 91125 \\ E-mail: rla@ipac.caltech.edu
}

\begin{abstract}
The structure and composition of circumstellar disks around young stars is one of the areas in which infrared interferometry has had a major impact. I will review the observational progress in this field starting from the earliest observations of $\mathrm{T}$ Tauri, Herbig $\mathrm{Ae} / \mathrm{Be}$ and FU Ori objects with smaller interferometers. Highlights of recent work include measurements of the inner disk size across 5 orders of magnitude in stellar luminosity, characterization of the dust composition as a function of radius in Herbig disks, high spectral resolution observations of gas emission and closure phase measurements of structure in disks. These observations have challenged models of disk structure and as the only direct probe on AU scales will continue to provide essential input in our understanding of the formation of stars and planets.
\end{abstract}

\section{Introduction}

The study of circumstellar disks around young stars has been one of the major areas of focus in infrared interferometry observations. One of the most exciting developments in the last few years is the expansion of the interferometers which can contribute to this area of study. I will attempt to touch on all aspects of YSO observations: disks, jets/winds and stellar properties. Other excellent reviews of this material exist, including Millan-Gabet et al (2007) in Protostars and Planets V. Some material for this work is taken from Akeson (2008a).

\subsection{Interferometers}

There are many current and recently operating optical and infrared interferometers (see reviews in the SPIE Proceedings vol. 6268, Advances in Stellar Interferometry). The results discussed here have come from the Palomar Testbed Interferometer (PTI), the Infrared Optical Telescope Array (IOTA), the Keck Interferometer (KI), the Very Large Telescope Interferometer (VLTI) and the Center for High Angular Resolution Astronomy (CHARA) array. The currently operating baseline lengths, wavelengths and website for more information on each of these arrays is given in Table 1 .

\subsection{Why infrared interferometry?}

The formation of low and intermediate mass stars involves a clump of gas and dust collapsing under the force of gravity to form a central star, a surrounding envelope, a flattened disk and outflowing material in a jet or wind. All of these components are not observable at every stage due to the high extinction of the envelope and surrounding cloud in the early stages and the low 
Table 1. The current operational capabilities of infrared interferometers discussed in this review.

\begin{tabular}{ll}
\hline Array name & Palomar Testbed Interferometer (PTI) \\
Baseline range/aperture diam (m) & $85-110 / 0.4$ \\
Wavelength bands & H,K \\
URL & http://msc.caltech.edu/missions/palomar/ \\
\hline Array name & Infrared Optical Telescope Array (IOTA) \\
Baseline range/aperture diam (m) & $15-40 / 0.4$ \\
Wavelength bands & H,K \\
URL & http://tdc-www.harvard.edu/IOTA/ \\
\hline Array name & Keck Interferometer (KI) \\
Baseline range/aperture diam (m) & $85 / 10$ \\
Wavelength bands & H,K,N \\
URL & http://planetquest.jpl.nasa.gov/keck \\
\hline Array name & Very Large Telescope Interferometer (VLTI) \\
Baseline range/aperture diam (m) & $8-200 / 1.8(A T), 8(U T)$ \\
Wavelength bands & J,H,K,N \\
URL & http://www.eso.org/projects/vlti/ \\
\hline Array name & Center for High Angular Resolution Astronomy (CHARA) \\
Baseline range/aperture diam (m) & $30-330 / 1$ \\
Wavelength bands & H,K \\
URL & http://www.chara.gsu.edu/CHARA/array.html \\
\hline
\end{tabular}

flux levels in the later stages. The star and disk are not observable in the optical and infrared until the $\mathrm{T}$ Tauri or Herbig Ae/Be phase when most or all of the envelope has been accreted or dispersed and it is on this stage that most of the infrared interferometry observations are concentrated. For high mass young stars most of the initial envelope must have dispersed before the young star can be observed at these wavelengths.

Infrared interferometry is well matched to the size scales and material temperatures in circumstellar disks. The nearest regions of ongoing star formation are 140 parsecs away. At this distance, $1 \mathrm{AU}$ subtends an angle of 7 milliarcseconds (mas). The fringe spacing (defined here as wavelength/baseline) for a 100 meter baseline is 4 mas at 2 microns and 20 mas at 10 microns. The fringe spacing is an indication of the largest diameter easily detectable on that baseline, as an object with a uniform disk angular diameter equal to the fringe spacing will have a visibility amplitude of 0.2 and a squared visibility amplitude of 0.04 .

Near-infrared wavelengths ( $\mathrm{H}$ and K, 1.6 and 2.2 microns respectively) are primarily sensitive to material emitting near $1000 \mathrm{~K}$, which corresponds to material in the inner disk, while midinfrared observations ( $\mathrm{N}$ band, 10 microns) are most sensitive to material at hundreds of Kelvin, corresponding to the middle disk and the habitable zone. Infrared interferometry observations are complimentary to observations at other wavelengths, particularly millimeter interferometry which probes the entire disk mass on scales from tens to hundreds of AU.

\section{Circumstellar disks}

Circumstellar disks around young stars are made up of several regions and the wavelengths and techniques used to observe these regions vary with the characteristic temperatures and size scales involved. In infrared interferometry, the near-infrared is used for the inner edge of the gas 
and dust disk, while the mid-infrared studies extended from the inner edge to several AU into the disk.

\subsection{Inner disk: dust and gas}

The questions to address using infrared interferometry in the inner disk include:

- What is the spatial and temperature distribution of gas and dust in the inner disk?

- Is there emission from another component on small scales?

The overall model in which most of these observations take place includes the central star, which for Herbig and T Tauri stars is a few solar radii and therefore unresolved, and the resolved disk whose visibility will depend on the size and flux profile. These disks often extend to $>100$ AU, but only the central few AU are warm enough to have significant emission at near-infrared wavelengths.

Classical models of geometrically flat accretion disks contain optically thick material all the way into the magnetospheric radius where the star's magnetic field lines truncate the disk. The disk is composed of gas and dust, except in the central region where the temperature is so high $(>1500 \mathrm{~K})$ that the dust sublimates. In this model, the predicted near-infrared visibility is very high as the magnetospheric radius is a few times the stellar radius (Figure 1). However, the initial observations with PTI and IOTA did not follow the predictions for T Tauri and Herbig stars. A survey of 15 Herbigs by Millan-Gabet et al (2001) found the resolved sources to be larger than predicted from an accretion disk with radial temperature profile $T \propto r^{-3 / 4}$ and no asymmetries observed on short to medium baselines. The first observations of $\mathrm{T}$ Tauri stars by Akeson et al (2000) found that disk emission from T Tau N and SU Aur came from radii larger than predicted from SED models. Interestingly, the very first YSO observed, FU Ori, did match the predicted structure [20].

At the same time, other techniques such as aperture masking were used to study even more massive YSOs. Imaging of $\mathrm{LkH} \alpha 101$ [37] revealed disk emission in an asymmetrically bright ring which the authors find to be consistent with the size expected for the sublimation point of optically thin dust. Several groups of modelers [30, 13, 29] proposed a solution to these observations and to the excess emission seen in the near-infrared SEDs of Herbig objects. In their physical models the central gas-only region is optically thin and therefore the dust sees the stellar flux directly. This direct heating results in the inner edge being vertically extended (Figure 1). These models can produce both the near-infrared bump seen in the SEDs of Herbigs, as the vertically extended inner rim has a larger emission region, and the resolved visibilities, as the near-infrared emission from the disk is dominated by inner rim, which is far enough from the central star to be resolved by interferometers with baselines of tens to a hundred meters.

Additional observations $[15,28,4]$ significantly expanded the sample of observed T Tauri and Herbig objects. Observations with longer baselines revealed the asymmetries expected for inclined disks $[15,5]$ but the overall trend of size corresponding to the dust sublimation radius held for the Herbig Be and T Tauri stars. However, the Herbig Ae stars seem consistent with the older geometrically thin but optically thick models (Figure 2). This difference could arise from a fundamental difference in the formation process of these higher mass stars or could simply be due to the different evolutionary state they are in when they become optically visible.

Recent studies have concentrated on examining the morphology of the inner rim in more detail and on determining if any gas exists within the dust sublimation radius. Akeson et al (2005a) used Monte Carlo scattering and emission models to fit the PTI and spectral energy distribution data of $3 \mathrm{~T}$ Tauri stars and found that the gas emission was significant for 2 of the objects. Pinte et al (2008) examined the interferometry data for all T Tauri observations and suggest that scattering (which will produced incoherent emission to the interferometers) may be an important component for the lowest mass T Tauris. Evidence for gas within the inner dust 


\section{Direct heating of inner dust disk}
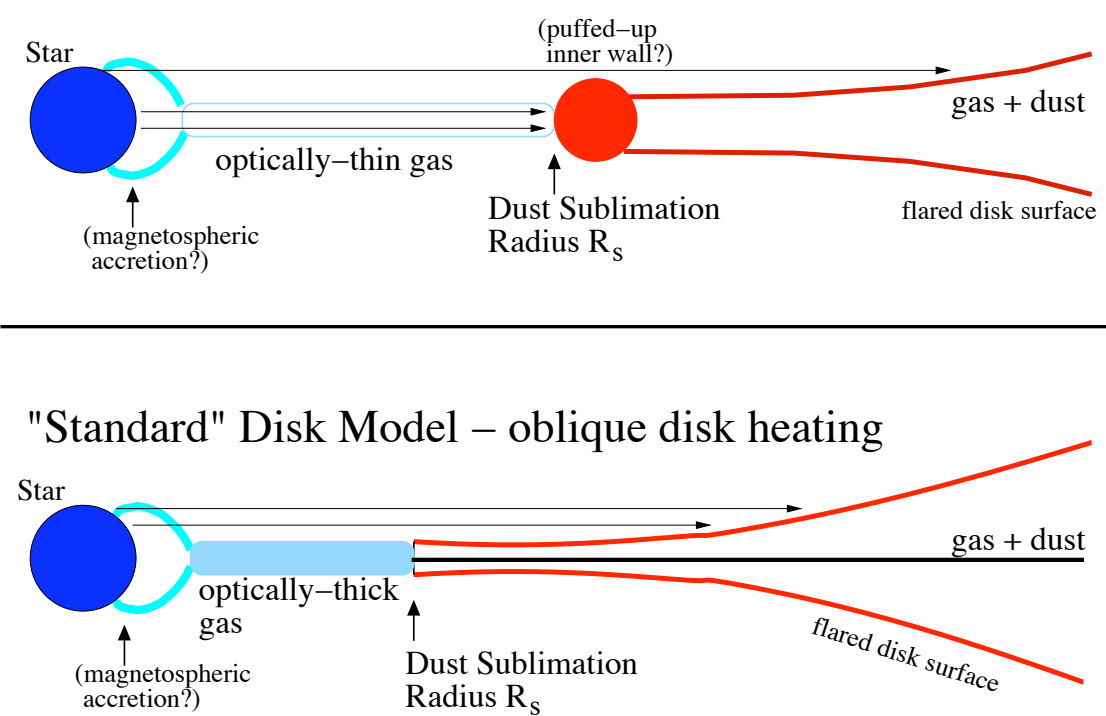

Figure 1. A schematic representation of the two models with optically thin material within the dust sublimation radius (top) and optically thick material to the magnetospheric radius (bottom). Figure taken from Millan-Gabet et al (2007).

radius is also inferred for Herbig stars by Kraus et al (2008) and Isella et al (2008) by combining observations across multiple bands. The shortest wavelengths are the most sensitive to the hot gas emission. Observations using very long baselines and closure phases have been used to study the detailed shape of the inner dust rim. Tannirkulum et al (2008) found that the data were best fit by very rounded inner dust rims.

The early studies all used continuum observations which are dominated by dust emission in the low to intermediate mass sources. Using higher spectral resolution $(\mathrm{R}>$ several hundred) it is possible to measure the visibility across spectral features in the near-infrared and directly compare the physical size of the gas-only region (line emission) to the gas and dust (continuum emission) region. Several other talks at this meeting (Kraus, van der Ancker) detail the results from these studies.

\subsection{Middle disk: composition and structure}

The middle disk (here roughly defined as 1 to tens of AU) has temperatures of several hundred Kelvin and is well probed by observations at mid-infrared wavelengths. Interferometry observations on 100 meter baselines will resolve structures on $1 \mathrm{AU}$ scales at the distance to the nearest star formation regions $(\mathrm{d} \sim 150 \mathrm{pc}$ ), but the more massive objects, particularly the Herbig Be stars are at much larger distances and the corresponding spatial resolution is lower. 


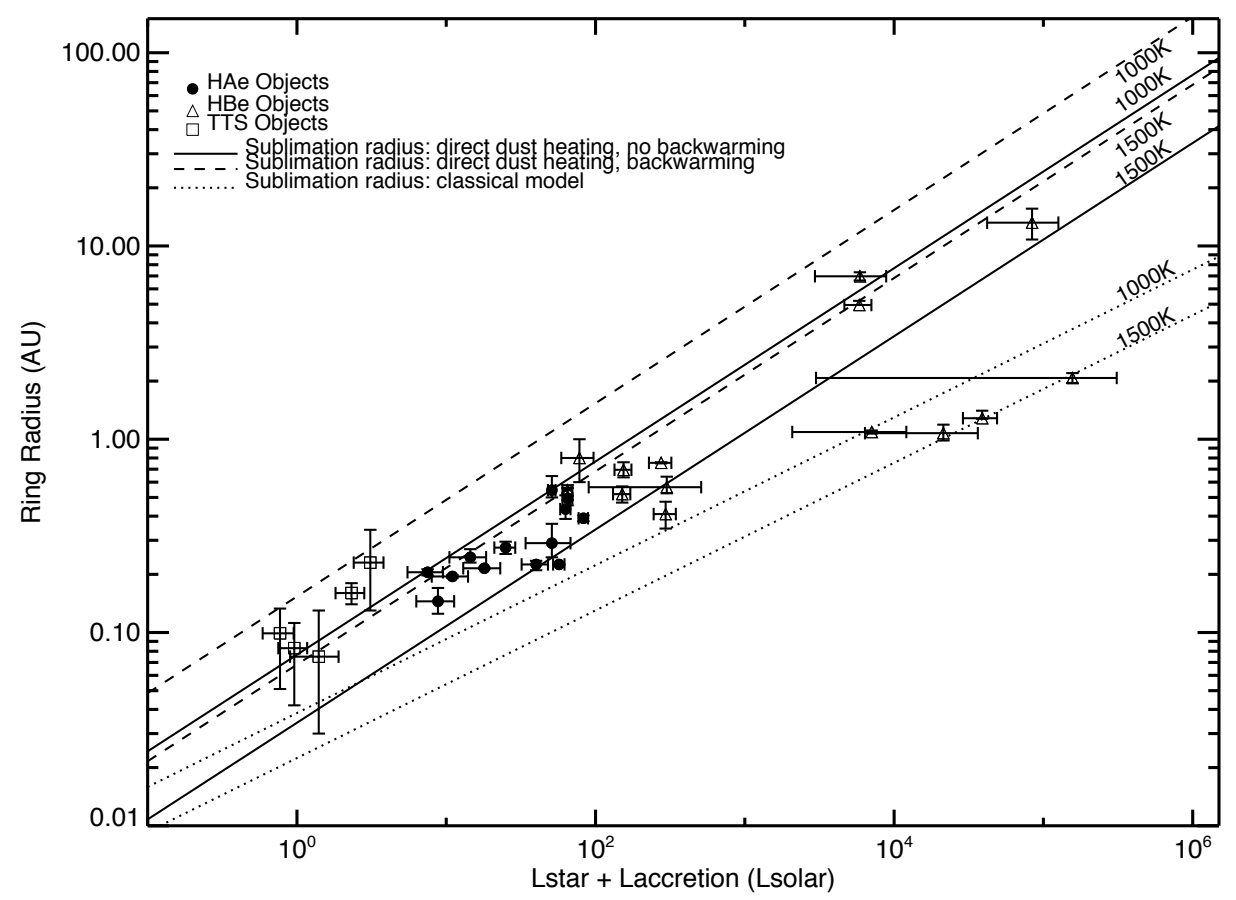

Figure 2. The measured sizes of Herbig Ae/Be and $\mathrm{T}$ Tauri stars vs the total stellar and accretion luminosity are plotted against sublimation radii for directly heated dust (solid and dashed lines) and for oblique heating (dotted line). Figure taken from Millan-Gabet et al 2007.

The majority of mid-infrared observations have been of Herbig Ae/Be stars with the MIDI instrument at VLTI. Figure 3 shows the measured half light radius in AU plotted against the mid-infrared color from a sample of objects observed by Leinert et al (2004). The most red object in mid-infrared colors (HD 100456) has a larger spatial radius than most of the more blue objects. These results are roughly consistent with the classification scheme proposed by Meeus et al (2001) of Group I flared disks, which appear larger and more red in the mid-infrared and Group II sources, which are self-shadowed and have a flatter geometric profile (see graphic at bottom of Figure 3). Leinert et al (2004) also find qualitative agreement between their results and the predictions of the disk models for these objects of Dominik et al (2003) which were fit only to the SEDs.

However, as more data are collected on a larger sample of sources and as some sources are considered in detail, exceptions to this classification scheme have been found. For example, Preibisch et al (2006) obtained MIDI/VLTI data on the Herbig A star HD 5999 and found visibilities much higher than those in the Leinert et al (2004) sample. In their models, the measured visibilities correspond to a physical size of 1 to $3 \mathrm{AU}$ and if the disk has a power-law density distribution, it must be truncated at $2-3 \mathrm{AU}$ in order to match the high visibility.

In addition to measuring the physical scales of the disk emission, the mid-infrared visibilities are generally spectrally dispersed with enough resolution to probe the composition of the disk as a function of radius. The mid-infrared region contains silicate features at $\sim 10$ microns, and so is ideal for studying the mineralogy. These results are discussed in this proceedings by van Boeckel. 

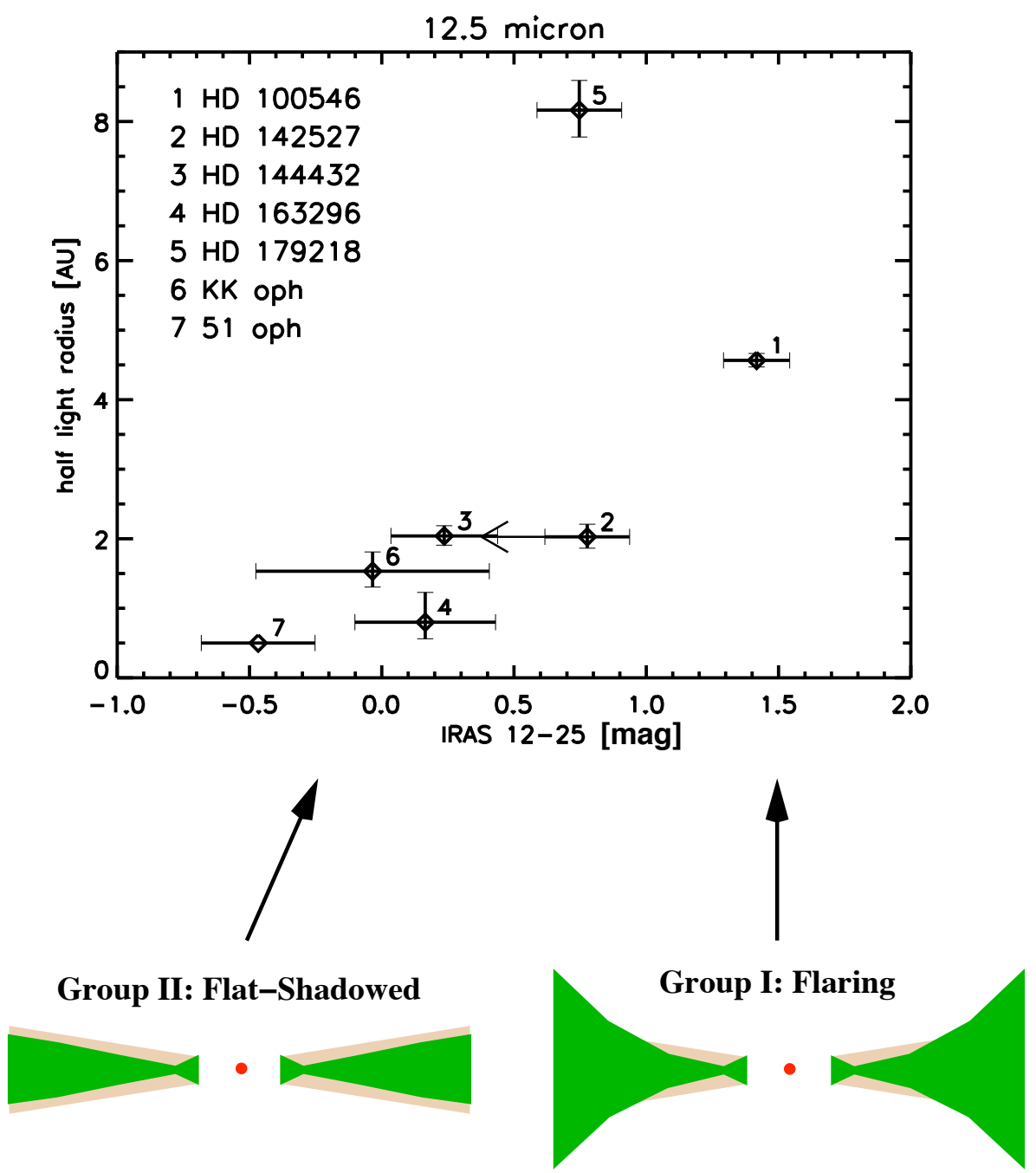

Figure 3. The relationship between the measured mid-infrared size and the infrared slope as measured from IRAS colors for a sample of Herbig stars observed with MIDI on the VLTI. The size-color relation is consistent with the SED classification of Meeus et al (2001). Figure taken from Millan-Gabet et al (2007).

\subsection{Disk summary}

Overall, the infrared interferometric observations of circumstellar disks show strong support for the inner dust radius being located at the dust sublimation radius and this has been shown to apply at luminosities from 1 to $\sim 10^{4} \mathrm{~L}_{\odot}$. At even higher luminosities, the disk emission is consistent with a optically thick disk extending to the magnetospheric radius. The mid-infrared observations follow the classification of Herbig Ae/Be sources into flared and self-shadowed morphologies and higher spectral resolution is tracing the dust composition as a function of radius.

\section{Jet and winds}

Increases in sensitivity and spectral resolution are allowing teams to investigate the jet and wind components of young stellar objects, which are though to be a ubiquitous part of star formation. 
In the first high resolution near-infrared observations, Malbet et al (2007) observed the disk and $\mathrm{Br} \gamma$ emission from massive young star MWC 297. The Br $\gamma$ emission clearly has a lower visibility than the continuum from the disk and the authors modelled this object with an optically thick disk and a latitude-dependent stellar wind outflow above the disk surface.

\section{Additional recent results}

Many other aspects of star formation are being examined using infrared interferometry. Some of these include:

- Observation of the older (10 Myr) transition disk around TW Hya at 2 and 10 microns find dust within the inner hole radius inferred from the spectral energy distribution [16, 34].

- Observations of the very massive young object W33A require a revised stellar luminosity [38].

- Observations of circumbinary disks have found evidence for disk warping in HD 98800 [6] and for emission inside the dynamical truncation radius in DQ Tau [11].

- Expanded near-infrared observations of the prototype FU Ori are consistent with an accretion disk only [21], while three other FU Oris require an extended flux component [26]. In the mid-infrared, V1647 Ori shows no spectral features, suggesting large dust grains [1] while FU Ori has weak, broad silicate features [31].

\section{Stellar properties}

A standard use of optical interferometers is the determination of binary physical orbits and stellar properties. The advent of large aperture interferometers has allowed this to extend to young stellar objects. This is particularly important for star formation models as there are few mass and luminosity determinations with sufficient accuracy to constrain models of pre-main sequence evolution. The first determination of YSO masses with long-baseline interferometry was on the southern binary component of the HD 98800 quadrapole system. Boden et al (2005) determined the masses with $10 \%$ uncertainties and these objects are among the lowest mass $\left(<0.7 \mathrm{M}_{\odot}\right)$ YSOs with dynamically determined masses. Additional systems are being studied $[10,35]$ and will constrain pre-main sequence evolutionary tracks.

\section{Debris disks}

The list of main sequence stars known to have circumstellar material in the form of debris disks has greatly expanded over the last few years from surveys at longer wavelengths and most recently from Spitzer observations [24]. Given the size and distribution of dust in these disks, it is generally believed that the dust is not remnant from the star formation process, but rather, is generated through collisions of larger bodies orbiting the star. These disks can extend over 1000 AU from the central star and may be analogs of our own solar system. Many of these systems are only a few hundred million years old and although the material does not have a primordial composition and or distribution, there are many similar aspects in the study of these disks and those around pre-main sequence objects.

Models of material in debris disk systems are a balance of collisions, radiation pressure, Poynting-Robertson drag and the dynamical influence of any large bodies in the system. In order to constrain these models, the dust spatial extent and grain size distribution must be measured. Observations of optical and near-infrared scattered light have provided the most detailed overall picture of the dust distribution. However, these scattered light observations do not have sufficient resolution to characterize the material closest to the star, and this is where infrared interferometry can provide a unique constraint. Spatially resolved observations in the near-infrared provide a much more sensitive probe of the inner regions of these disks. Both PTI 
[12] and CHARA [2] have detected near-infrared extended emission around Vega, the prototype debris disk system, indicating a $2 \%$ excess above the stellar photosphere. While a near-infrared excess was not known through broadband spectral modelling, the interferometrically detected near-infrared excess is within the photometric uncertainties. This technique relies on precise measurement of the visibility to measure the departure from the stellar size. An example data set on the debris disk system $\beta$ Leo is shown in Figure 4. Note that these objects are generally much closer than the nearest YSO's and so the star itself is resolved. The stellar size is determined by the long baseline measurements, and when this is extrapolated to smaller spatial frequencies (shorter baselines), it is clear that the visibility is lower than expected. The data are consistent with an extended (i.e., over-resolved) emission component, which due to the temperatures sampled at 2 microns and the field-of-view, must be at smaller spatial scales than the cooler material which produces the previously known mid-infrared excess toward this star [8].

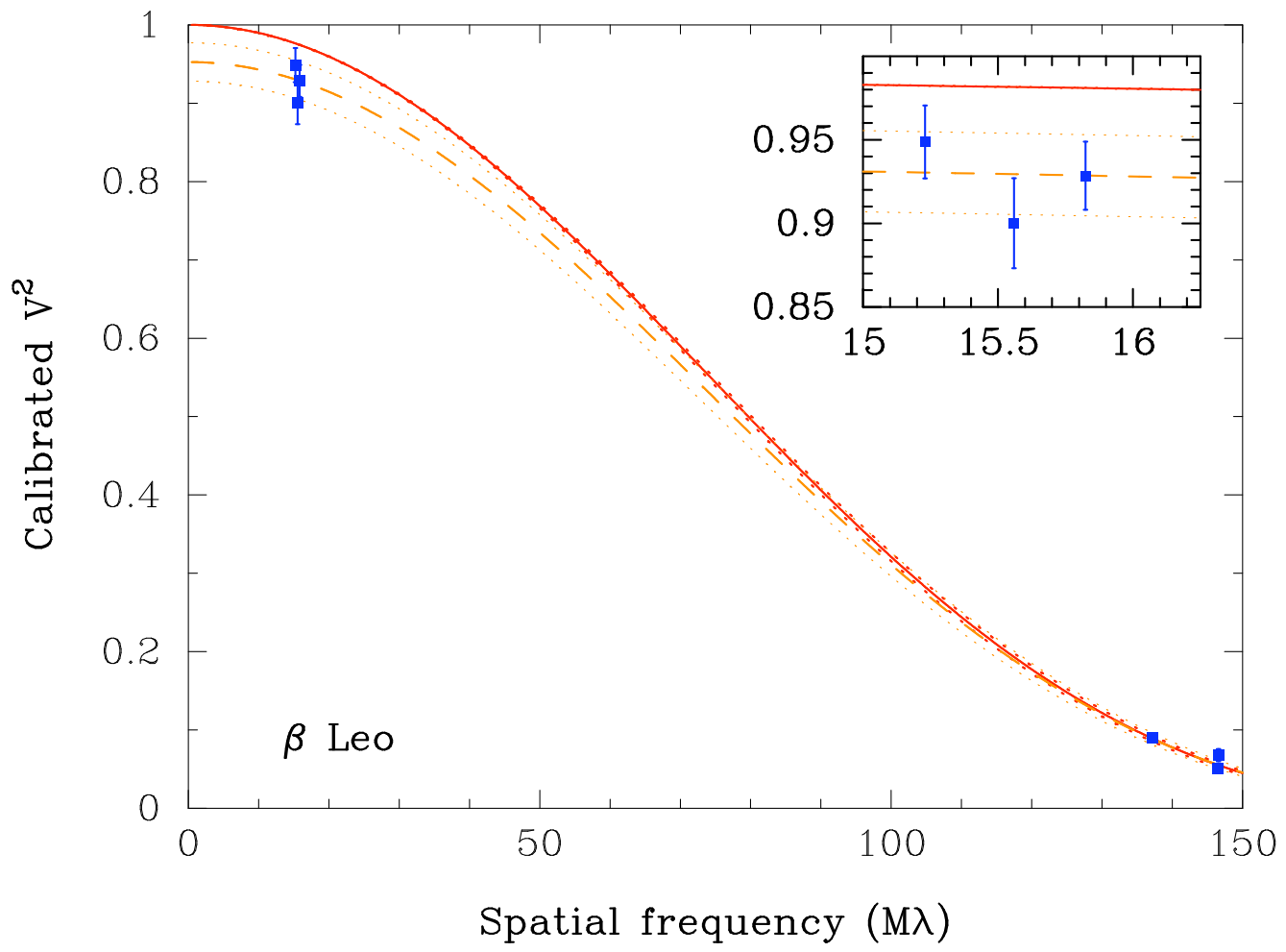

Figure 4. The measured visibility for $\beta$ Leo from CHARA [8]. The red line solid line (with errors) shows the uniform disk fit to the higher spatial frequencies, which does not fit the lower spatial frequencies. The orange dashed line (with errors) shows the fit using a uniform disk and incoherent emission component.

\section{Conclusions}

The capabilities of infrared interferometers are still rapidly improving and even more exciting discoveries are sure to be made in the next few years. KI is adding L-band $(3 \mu \mathrm{m})$ and phasereferencing to extend sensitivity by several magnitudes and is performing a $10 \mu \mathrm{m}$ survey of nearby main sequence stars for exozodiacal dust. CHARA has imaging capabilities with MIRC and will add improved sensitivity with fringe tracking. The VLTI second generation instruments 
VSI, MATISSE and GRAVITY all have applications for young stars. And two facilities under construction, LBTI and MROI will also be useful for these sources.

Star formation is arguably the area of astrophysics in which infrared interferometry has had the biggest impact. The main discovery is that the optically thick portion of T Tauri and Herbig $\mathrm{Ae} /$ Be disks DO NOT extend to a few stellar radii of the stellar surface. While observations show that emission is coming from near the dust sublimation radius, it is not all from a single radius, leading to further questions: What is the exact structure of the dust sublimation boundary? How does the inner disk evolve with time? How does this effect planet formation and migration?

The observational prospects are rapidly improving and higher spectral resolution allow observations of the gas: jets, winds, accretion, while closure phase and imaging will help eliminate model uncertainties and dependencies.

\section{References}

[1] Ábrahám P, Mosoni L, Henning Th, Kóspál Á, Leinert Ch, Quanz S P and Ratzka Th 2006 A $\& A 449$ L13

[2] Absil O, di Folco E, Mérand A, Augerau J-C, Coudé du Foresto V, Aufdenberg J P, Kervella P, Ridgway S T, ten Brummelaar T A and McAlister H A 2006 A $\mathscr{U} A 452237244$.

[3] Akeson R L, Ciardi D R, van Belle G T, Creech-Eakman M J and Lada E A 2000 ApJ 543313.

[4] Akeson R L, Boden A F, Monnier J D, Millan-Gabet R, Beichman CBeletic J, Calvet N, Hartmann L, Hillenbrand L, Koresko C. et al. 2005 ApJ 6351173

[5] Akeson R L, Walker C H, Wood K, Eisner J A and Scire E 2005 ApJ 622440

[6] Akeson R L, Rice W K M, Boden A F, Sargent A I, Carpenter J M and Bryden, G 2007 ApJ 6701240

[7] Akeson R 2008, New Astronomy Review 5294

[8] Akeson R et al. 2008 ApJ submitted

[9] Boden A F, Sargent A I, Akeson R L, Carpenter J M, Torres G, Latham D W, Soderblom D R, Nelan E, Franz O G and Wasserman L H 2005 ApJ 635442

[10] Boden A F, Torres G, Sargent A, Akeson R L, Carpenter J M, Boboltz D A, Massi M, Ghez A M, Latham D W, Johnston K J et al. 2007 ApJ 6701214

[11] Boden A F, et al. $2008 A p J$ in press

[12] Ciardi D R, van Belle G T, Akeson R L, Thompson R R and Lada E A 2001 ApJ 5591147

[13] Dullemond C P, Dominik C and Natta A 2001 ApJ 560957

[14] Dominik C, Dullemond C P, Waters, L B F M and Walch S 2003 A $\& A 398607$

[15] Eisner J A, Lane B F, Hillenbrand L A, Akeson R L and Sargent A I 2004 ApJ 6131049

[16] Eisner J A, Hillenbrand L A, White R J, Akeson R L, and Sargent A I 2005 ApJ 623952

[17] Kraus S, Preibisch T and Ohnaka K 2008 ApJ 676490

[18] Isella A, Tatulli E, Natta A and Testi L 2008 A 6 A 483 L13

[19] Leinert Ch et al. $2004 A \mathscr{G} A 423537$

[20] Malbet $\mathrm{F}$ et al. 1998 ApJ $\mathbf{5 0 7}$ L149

[21] Malbet $\mathrm{F}$ et al. $2005 A \& A 437627$

[22] Malbet $\mathrm{F}$ et al. $2007 A \& B 46443$

[23] Meeus G et al. 2001 A\&S $36 \mathbf{6 5}$ 476-490

[24] Meyer M R, Backman D E, Weinberger A J and Wyatt M C 2007 Protostars and Planets V 573

[25] Millan-Gabet R, Schloerb F P and Traub W A 2001 ApJ 546 358-381

[26] Millan-Gabet R, Monnier J D, Akeson R L, Hartmann L, Berger J-P, Tannirkulam A, Melnikov S, Billmeier R, Calvet N, D'Alessio P et al. 2006 ApJ 641 547-555

[27] Millan-Gabet R, Malbet F, Akeson R, Leinert C, Monnier J and Waters R 2007, Protostars and Planets V 539

[28] Monnier J D, Millan-Gabet R, Billmeier R, Akeson R L, Wallace D, Berger J-P, Calvet N, D'Alessio P, Danchi W C, Hartmann L, et al. 2005 ApJ $\mathbf{6 2 4} 832-840$

[29] Muzerolle J, Calvet N, Hartmann L and D'Alessio P 2003 ApJL 597 L149

[30] Natta A, Prusti T, Neri R, Wooden D, Grinin V P et al. $2001 A \mathscr{E} A 371$ 186-197

[31] Quanz S P, Henning T, Bouwman J, Ratzka T and Leinert C 2006 ApJ 648472

[32] Pinte C, Ménard F, Berger J P, Benisty M and Malbet F 2008 ApJL 673 L63

[33] Preibisch T, Kraus S, Driebe T, van Boekel R and Weigelt G 2006 A 6 A 458235

[34] Ratzka T, Leinert C, Henning T, Bouwman J, Dullemond C P and Jaffe W 2007 Astronomische Nachrichten 328651

[35] Schaefer G H, Simon M, Prato L and Barman T 2008 AJ 1351659

[36] Tannirkulam A, et al. 2008 ApJL 677 L51 
[37] Tuthill P G, Monnier J D, Danchi W C, Hale D D S and Townes C H 2002 ApJ 577826

[38] de Wit W J, Hoare M G, Oudmaijer R D and Mottram J C 2007 ApJL 671 L169 